\title{
Gotthold Eisenstein and Philosopher John
}

Franz Lemmermeyer (Gymnasium St. Gertrudis in Ellwangen, Germany)

Before the recent publication of the correspondence between Gauss and Encke, nothing was known about the role that John Taylor, a cotton merchant from Liverpool, had played in the life of Gotthold Eisenstein. In this article, we will bring together what we have discovered about John Taylor's life.

\section{Eisenstein's journey to England}

Gotthold Eisenstein belonged, together with Dirichlet, Jacobi and Kummer, to the generation after Gauss that shaped the theory of numbers in the mid-19th century, and like Galois, Abel, Riemann, Roch and Clebsch, Eisenstein died young. Today, Eisenstein's name can be found in the Eisenstein series, Eisenstein sums, the Eisenstein ideal, Eisenstein's reciprocity law and in his irreducibility criterion, and he is perhaps best known for his ingenious proofs of the quadratic, cubic and biquadratic reciprocity laws. Eisenstein's father Johann Constantin Eisenstein emigrated to England in 1840; Eisenstein and his mother followed in June 1842, although Eisenstein's few remarks on this episode in his autobiography [3] belie the dramatic events that he experienced in England. On their journey to England, the Eisensteins passed through Hamburg; during the Great Fire in May 1842 about a third of the houses in the Altstadt had burned down. What we learn from Eisenstein's account is that he was impressed by the sight of railroad lines running right under the foundations of houses (in London?) and by the Menai suspension bridge in Wales: Eisenstein mentions that he undertook six sea voyages, and that on one of them they sailed under the tremendous suspension bridge in Anglesey, which was so high that the Berlin Palace would easily have fitted under its main arch.

Eisenstein also writes that he later made the acquaintance of William Hamilton in Dublin (who would discover the quaternions in October 1843), as well as that of the mayor of Dublin, Daniel O'Connell (who died in 1847 in

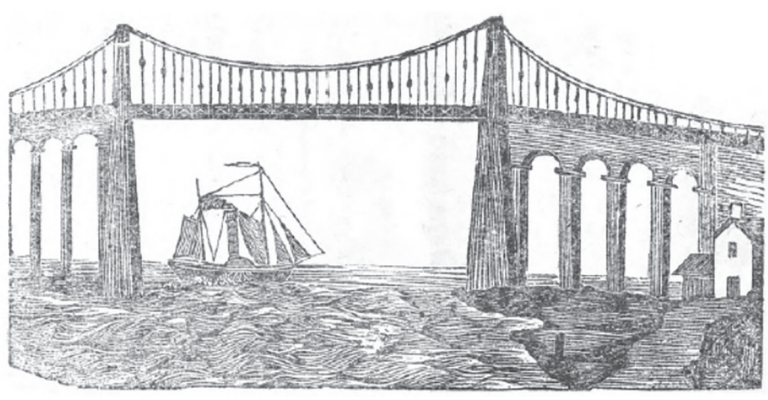

Menai suspension bridge (from [1]).
Genoa, where he wanted to organise help for the Irish during the Great Famine).

Eisenstein does not mention what happened after his arrival in England; the correspondence between Encke and Gauss reveals that he fell seriously ill and was saved by the assistance of a certain Mr. Taylor from Liverpool. Encke first mentions this story in his letter from 10 June 1844 (see [10], p. 1141):

I have the honour, most esteemed Privy Counsellor, to send you the second volume of the Berlin Observations, and I have enclosed a collection of papers by a young local mathematician Eisenstein, whose assessment seems to me to be of importance in the interest of science, and for which no better judge could ever be found than you, the true head of this part of higher mathematics. I would not have concerned myself with this matter, but would have left it to Prof. Dirichlet, if the latter had not already been away for a year.

Young Eisenstein went to the same Gymnasium as my sons, where he did not excel at other subjects, but surprised his teacher in mathematics ${ }^{1}$ with a curious derivation of a series first developed by Lagrange, without having known this paper. He was then introduced to Prof. Dirichlet, who believed him to be an extraordinary genius. Owing to his family being in special financial circumstances, he followed his parents to Liverpool two years ago, where illness and family relations (his father seems to have speculated unsuccessfully) reduced him to such a harassed state that his local acquaintances thought it necessary to send him back home. Dirichlet told me of these circumstances and asked me to try to aid his return with the help of my English friends. Although I did not have any acquaintances in Liverpool, it turned out that an important cotton merchant, Taylor, had submitted a book on ancient Roman festivities and chronology to the Academy, and I learned that he published articles in journals on our comet and in particular on the short-period comet. ${ }^{2}$ Based on this, I turned to this gentleman, who was otherwise completely unknown to me, and asked for his assistance. With a generosity that can perhaps only be found in Englishmen, he sought out young Eisenstein, arranged for a doctor and medicine (Eisenstein was suffering from typhoid fever), provided him with the means for travelling to Dublin (where Hamilton received him very obligingly) and also gave

1 This might have been Karl Heinrich Schellbach.

2 Encke had computed the elements of the comet now named after him; it has an orbital period of only 3.3 years. 
him and his mother the necessary funds to return to Berlin. Here, Eisenstein occupied himself with investigations of the kind you will find among the enclosed papers and has obtained, since other avenues were unsuccessful, a yearly sum of money from the King (through the efforts of Mr.v. Humboldt, as he told me), with which this young man is completely content (in his own words). He would like to visit Göttingen in the near future in order to present himself to you and, if the circumstances allow, profit from your teaching.

Because of his young age (he cannot yet be or must only just be 20) and his talent, which he certainly must possess, although I admittedly cannot tell whether it is as great as Dirichlet's remarks would have one believe, your judgement would be of such importance for his future position that I entreat you with some urgency not to deny my request. He seems to me to be pleasant company, and his experiences do not appear to have weighed him down, but rather shown him that he must pull himself together, and I believe I may hope that he will not be a burden to you. The manner in which I am involved in these matters is not entirely comfortable to me, since I feel indebted to Mr. Taylor and must see how I can thank him.

Gauss answers Encke's letter on 23 June and writes:

I would be pleased to make the acquaintance of such a promising young man, and I would be delighted [...] if he would spend some time in G[öttingen]. It would be my pleasure if I could be of any assistance to him, if not by actual teaching, since he clearly has by far surpassed this stage.

Gauss's judgement on Eisenstein's skills was based on the articles that Eisenstein published in Crelle's Journal in early 1844. Gauss had only studied one of these articles in detail, namely Eisenstein's proof of the quadratic reciprocity law using multiple Jacobi sums (see [3], vol. I, pp. 100-107), but this was sufficient to convince Gauss of Eisentein's talent.

On 13 August 1844, Encke writes to Gauss, asking him to reply to a letter from Taylor and reminding him what Taylor has done for Eisenstein:

Young Eisenstein, in whom you have shown so much interest, went to Liverpool in early 1843 (unless I'm mistaken) with his (rather worthless) father; there his family got into a serious plight, and in addition young Eisenstein became gravely ill with typhoid fever. Prof. Dirichlet told me about it and asked if I happened to have an acquaintance in Liverpool who could take an interest in the young man, for otherwise he would perish.

Although I did not know Mr. Taylor (I had only heard from Prof. Mitzscherlich that he was a wealthy cotton merchant and an amateur astronomer who pub-

\footnotetext{
3 Eilhard Mitzscherlich (1799-1863) was a chemist and mineralogist from Berlin.
}

lished news about comets and was familiar with my name), I took the chance of approaching him about the matter. He immediately did a lot more than I had hoped, sending a doctor to young Eisenstein, providing for him by a subscription, supplying him with money for travelling to Dublin in order to meet Hamilton, and facilitating his return to Berlin.

\section{John Taylor}

Who was this Mr. Taylor, cotton merchant from Liverpool, who saved Eisenstein's life and paid for his travels to Ireland and back to Germany? Encke writes in a letter to Gauss from 15 August 1846:

I must confess that I think very highly of his actions, for which he received no compensation, and so I am sorry that he got into a bitter dispute with Sheepshanks concerning the building of the observatory, in which he (probably deservedly) drew the short straw, since he does not know much about modern astronomy. He knows more about ancient astronomy, since he has translated the first four books of Ovid's fastis and published them with explanations about the knowledge of the skies at that time.

Should you thus feel inclined to answer his letter, I would sincerely request that you make friendly mention of his truly noble behaviour towards young Eisenstein, which he would value highly.

The information that we can glean from Encke's letter suffices to identify Taylor as the cotton merchant John Taylor from Liverpool; both his Poems and Translations, including the English translation of Ovid's fastis [9], and his heated exchanges with Reverend Sheepshanks in [7] can be found online.

Richard Sheepshanks (1794-1855) had been a Fellow of the Royal Society in London since 1830. The list of people he quarreled with is long; his dispute with James South, which Charles Babbage and his difference engine got drawn into later, is described in Hoskin's article Astronomers at War [5].

The dispute with Taylor was about the best position for the future obervatory in Liverpool. In a letter to the Liverpool Mercury, Taylor heaps scorn upon Sheepshanks' choice:

according to the writers of this Royal Astronomical Report, the proper situation for our Observatory is in the lowest point of land that can be found, surrounded by hills that cut off the true horizon, and where, in fact, there is no horizon at all, although, to be sure, when the trough of the river is filled with smoke and fog, which is commonly the case, there may seem to be a horizon at a few yards distance [...] where nor sun, nor moon, nor star is ever seen, or was ever seen, to rise or set, and where no meridian line can ever be drawn or determined [...].

Sheepshanks prevailed, but 20 years later the observatory was closed due to an extension of the harbour, and 
in 1868 the new observatory was erected on Bidston Hill, the position that had been suggested by Taylor.

Another source of information on John Taylor comes from the diary of the American astronomer Maria Mitchell. In 1857 she travelled through Europe; on 3 August 1857 (see [6], p. 86) she delivered a letter to John Taylor and observed that he must have been around 80:

I brought a letter from Professor Silliman to Mr. John Taylor, cotton merchant and astronomer; and to-day I have taken tea with him. He is an old man, nearly eighty I should think, but full of life, and talks by the hour on heathen mythology. He was the principal agent in the establishment of the Liverpool Observatory, but disclaims the honor, because it was established on so small a scale, compared with his own gigantic plan. Mr. Taylor has invented a little machine, for showing the approximate position of a comet, having the elements. [...] He struck me as being a man of taste, but of no great profundity.

If Taylor was about 80 years old in 1857, then he must have been born around 1777 . His cometarium was studied by Beech [2]. The information that Taylor died in 1857 can be found in [4], p.191:

One of Mr. William Ewart's strongest political supporters during the stirring times which preceded the passage of the first Reform Bill (of which Mr. Ewart became an energetic advocate) was Mr. John Taylor, who, along with his brother Richard, had commenced business as cotton broker in 1821, but who since 1826 had been by himself. He was commonly called "Philosopher John”, for besides being an active politician, both as writer and speaker, he was also noted as a poet and as an astronomer. He was the first to propose the erection of an Observatory in Liverpool, and out of this suggestion originated the present establishment at Bidston Hill. He was one of the original members of the Cotton Brokers' Association, and continued in business until his death, which occurred in 1857.

William Ewart (1798-1869) was a liberal politician from Liverpool fighting for the abolition of capital punishment; he voted for the legalisation of the metric system in England in 1864.

\section{References}

[1] A description of the grand suspension bridge, L.E. Jones 1842.

[2] M. Beech, The Cometarium by John Taylor, Bull. Scientic Instrument Society 88 (2006), 2-6.

[3] G. Eisenstein, Mathematische Werke, 2 Bände, Chelsea 1975.

[4] Th. Ellison, The cotton trade of Great Britain, London 1886.

[5] M. Hoskin, Astronomers at war: South vs. Sheepshanks, J. Hist. Astr. 20 (1989), 175-212.

[6] Ph. Mitchell Kendall (Hrsg.), Maria Mitchell. Life, letters, and journals, Boston 1896.

[7] Rev. R. Sheepshanks, Correspondence respecting the Liverpool observatory between Mr. John Taylor and the Rev. R. Sheepshanks, 1845.

[8] Rev. R. Sheepshanks, A letter to the Board of visitors of the Greenwich royal observatory in reply to the calumnies of Mr. Babbage at their meeting in June 1853, and in his book entitled The exposition of 1851, London 1860.

[9] J. Taylor, Poems and Translations, Liverpool 1839

[10] A. Wittmann, Obgleich und indeßen: Der Briefwechsel zwischen Carl Friedrich Gauß und Johann Franz Encke, Kessel Verlag 2018.

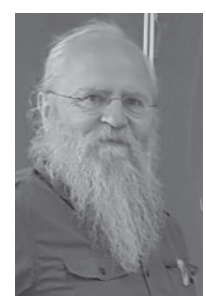

Franz Lemmermeyer is teaching mathematics at the Gymnasium St. Gertrudis in Ellwangen. He is interested in number theory and the history of mathematics. 